\title{
Value of Neutrophil:Lymphocyte Ratio Combined with Sequential Organ Failure Assessment Score in Assessing the Prognosis of Sepsis Patients
}

\author{
Yixuan Li, Junyu Wang, Bing Wei, Xiangqun Zhang, Le Hu, Xinghua Ye (D) \\ Emergency Medicine Clinical Research Center, Beijing Chao-Yang Hospital, Capital Medical University, Beijing, People's Republic of China \\ Correspondence: Junyu Wang; Bing Wei, Email wangjunyul007@I63.com; dr_weibing@I26.com
}

Background: We aimed to evaluate risk factors and assessment values in patients with sepsis and to explore a method of improving prognosis-prediction efficiency for patients with sepsis.

Methods: Patients with sepsis admitted to the Emergency Medicine Clinical Research Center, Beijing Chao-Yang Hospital, Capital Medical University from January 2020 to December 2020 were enrolled. Demographic data of patients and laboratory values at admission were collected. Sequential organ failure assessment (SOFA) and acute physiology and chronic health evaluation II (APACHE II) scores were calculated within 24 hours of admission and logistic regression used to analyze risk factors of death within 28 days, as well as the combined predictor of SOFA score and neutrophil:lymphocyte ratio (NLR). Predicted values of various indicators for 28-day mortality in sepsis patients were analyzed using receiver-operating characteristic curves.

Results: A total of 302 patients were included in this study, of whom 64 (21.2\%) died. Age, Pct, CRP, Lac, NLR (OR 1.054, 95\% CI 1.032-1.076), SOFA score (OR 1.434, 95\% CI 1.293-1.591), APACHE II score (OR 1.231, 95\% CI 1.166-1.300), and NLR combined with SOFA (SOFA+NLR $\times 0.149$ : OR $1.455,95 \%$ CI 1.318-1.605) were risk factors of 28-day mortality in sepsis patients, and areas under the curve of NLR combined with SOFA score were significantly higher than each of NLR and SOFA scores and similar to APACHE II scores. The sensitivity and specificity of NLR combined with SOFA and APACHE II scores to predict the 28-day prognosis of sepsis patients were better than the other indicators.

Conclusion: NLR combined with SOFA was a risk factor of the death of sepsis patients and its predictive efficacy similar to that of the APACHE II score, which is superior to other predictive indices.

Keywords: sepsis, risk factor, neutrophil to lymphocyte ratio, SOFA, APACHE II

\section{Introduction}

Sepsis is a life-threatening organ-dysfunction syndrome due to a disordered response of the body to infection. ${ }^{1}$ There are approximately 31.5 million patients contracting sepsis and 19.4 million septic shock worldwide each year, of which 5.3 million die, with an in-hospital mortality rate of $17 \%-31 \% .^{2,3}$ The high in-hospital mortality rate of septic shock patients makes sepsis one of the most severe problems faced by medical workers in clinical work globally. Acute physiology and chronic health evaluation II (APACHE II) scores are used to assess outcomes in intensive care units. Kumar et $\mathrm{al}^{4}$ found that APACHE-II scores had sensitivity of $89.9 \%$ and specificity of $97.6 \%$ in predicting death. However, APACHE II grading is based on 12 physiological indices (the worst value admitted to hospital within 24 hours), age, and chronic health conditions to assess the severity of disease and needs lots of clinical data and laboratory tests, rendering it too complicated to obtain a specific rating initially and thus limiting its clinical application. In order to effectively improve the prognosis of patients with sepsis and the survival rate in China, determining the severity and prognosis of patients with sepsis quickly and correctly is crucial. The aim of this study was to investigate a fast and accurate combination of indicators for predicting death within 28 days in patients with sepsis. 


\section{Methods}

\section{Participants}

This retrospective study was conducted on sepsis patients admitted to the Emergency Medicine Clinical Research Center, Beijing Chao-Yang Hospital, Capital Medical University from January 2020 to December 2020.

\section{Inclusion Criteria}

Patients aged $>18$ years with nonsurgical trauma, had survived in the emergency room $\geq 24$ hours, met the definition and diagnostic criteria of Sepsis-3 published by the American Society for Critical Care Medicine and the European Society for Critical Care Medicine in 2016, ${ }^{1}$ and had been clinically diagnosed as having sepsis by two senior physicians were involved in this investigation.

\section{Exclusion Criteria}

Patients who did not meet Sepsis-3 diagnostic criteria, had incomplete data within 24 hours of admission, had survived $<24$ hours in the emergency room, were pregnant, had immune-system diseases, malignant tumors, or hematological diseases, or were on glucocorticoids were excluded. Data on first admission were obtained for patients who visited the hospital repeatedly during the inclusion period.

\section{Sample-Size Calculation}

We used a sample-size calculation formula for a cross-sectional study of classification variables: $\mathrm{n}=\left(Z_{1-\alpha / 2} / \delta\right)^{2} \times p \times(1-p)$, $Z_{1-\alpha / 2}=1.96, \delta=0.05, p=0.25$ (based on mortality in patients with sepsis reported in the literature of about $25 \%{ }^{2,3}$ ). Therefore, the total sample size of the study needed to be at least 288 cases.

\section{Data Collection}

Age, sex, pulse, blood pressure, and consciousness status of sepsis patients were collected at admission, as well as levels of CRP, procalcitonin (Pct), white blood cells (WBCs), absolute neutrophil count, absolute lymphocyte count, absolute platelet count, creatinine, urea nitrogen, and lactic acid (Lac). Based on initial data obtained within the first 24 hours, sequential organ failure assessment (SOFA) scores, acute physiology and chronic health assessment II (APACHE II) scores, and neutrophil:lymphocyte ratios (NLRs) were calculated. Death or survival within 28 days was recorded.

\section{Statistical Analysis}

SPSS 22.0 was used for data analysis. Data conforming to normal distribution are represented by means $\pm \mathrm{SE}, \bar{X}$ abnormal distribution by quartiles, and count data by percentages. Student's $t$-test was used for parameters that fit normal distribution, nonparametric tests for abnormal distribution, and $?^{2}$ tests for comparison of rates. Logistic regression was used to analyze risk factors of death within 28 days. Predictors of NLR combined with SOFA were calculated via a formula predictor - Mark1+Mark2 $\times(\mathrm{B} 2 / \mathrm{B} 1)$ - where B1 and B2 were the constants of the logistic regression of Mark1 and Mark2, respectively. Receiver-operating characteristic (ROC) curves were plotted and corresponding 95\% CIs, area under the curve (AUC), sensitivity, specificity, and optimal cutoff calculated. The $Z$ test was used

to compare the AUC between the indicators $\left(Z=[\mathrm{A} 1-\mathrm{A} 2] / \sqrt{\mathrm{SE}_{1}^{2}+\mathrm{SE}_{2}^{2}}, Z_{0.05}=1.96, Z_{0.01}=2.58, Z>Z_{0.05}\right.$ means $\left.P<0.05\right)$. $\alpha=0.05$ was used as the test level, and $P<0.05$ was considered significant.

\section{Results}

\section{General Information}

A total of 302 patients were involved in this study (Figure 1), of whom 64 (21.2\%) died. Demographic and clinical data of the two groups are compared in Table 1. Age, pulse, consciousness disorder, Pct, CRP, WBCs, neutrophils, creatinine, urea nitrogen, Lac, NLR, SOFA scores, and APACHE II scores were significantly higher in the death group than the survival group $(P<0.001)$. Similarly, blood pressure and lymphocytes were much lower in the death group than the survival group. There were no significant differences in sex or platelets between the groups $(P>0.05)$. 


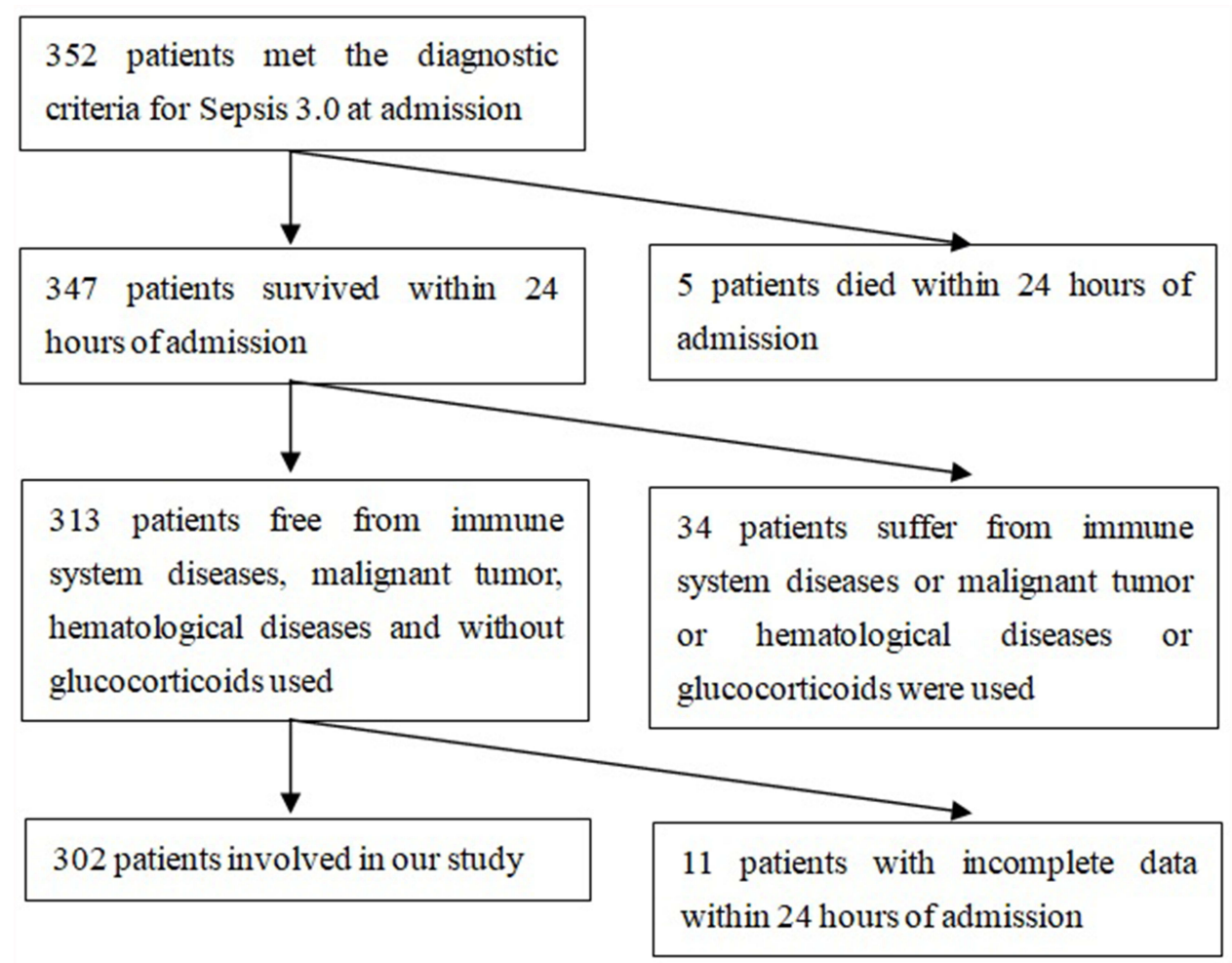

Figure I Flow chart for patients with sepsis.

\section{Risk Factors of 28-Day Mortality in Patients with Sepsis}

The 28-day survival status of patients with sepsis was used as the dependent variable, and age, Pct, CRP, Lac, NLR, SOFA score, and APACHE II score were used as independent variables to perform logistic regression analysis (Table 2). Age, Pct, CRP, Lac, NLR, SOFA score, and APACHE II score were found to be risk factors of 28-day mortality in sepsis

Table I Demographic and clinical data

\begin{tabular}{|c|c|c|c|}
\hline & Survival group $(n=238)$ & Death group $(n=64)$ & $P$ \\
\hline Age (years) & $72 \pm 14$ & $78 \pm 10$ & $<0.001$ \\
\hline Sex (male) & I33(55.9\%) & $42(65.6 \%)$ & 0.161 \\
\hline Pulse (beats/minute) & $94 \pm 24$ & $101 \pm 28$ & 0.113 \\
\hline Systolic pressure $(\mathrm{mmHg})$ & $138 \pm 27$ & $118 \pm 35$ & $<0.001$ \\
\hline Diastolic pressure $(\mathrm{mmHg})$ & $74 \pm 17$ & $63 \pm 20$ & $<0.001$ \\
\hline Consciousness disorder (\%) & $17.0 \%$ & $50.8 \%$ & $<0.001$ \\
\hline Pct $(n g / m L)$ & $0.05(0.5,0.27)$ & $1.00(0.09,5.95)$ & $<0.001$ \\
\hline CRP (mg/L) & $14.0(8.0,65.8)$ & $93.0(43.3,120.0)$ & $<0.001$ \\
\hline WBCs $\left(\times 10^{9} / L\right)$ & $8.90(6.48,11.70)$ & $11.25(8.03,16.60)$ & $<0.001$ \\
\hline Neutrophils $\left(\times 10^{9} / \mathrm{L}\right)$ & $6.86(4.70,9.48)$ & $9.69(6.43,14.58)$ & $<0.001$ \\
\hline Lymphocytes (× $\left.10^{9} / L\right)$ & $1.16(0.73,1.70)$ & $0.96(0.58,1.37)$ & 0.042 \\
\hline Platelets $\left(\times 10^{9} / \mathrm{L}\right)$ & $185(142,256)$ & $203(129,282)$ & 0.409 \\
\hline Creatinine $(\mu \mathrm{mol} / \mathrm{L})$ & $81(62,119)$ & $127(86,253)$ & $<0.001$ \\
\hline Urea nitrogen (mmol/L) & $7.17(5.05,10.87)$ & $15.09(8.70,23.75)$ & $<0.001$ \\
\hline Lac $(\mathbf{m m o l} / \mathbf{L})$ & $1.20(0.80,1.80)$ & $2.20(1.50,4.35)$ & $<0.001$ \\
\hline NLR & $6.25(3.18,11.33)$ & $|2.4|(6.45,28.32)$ & $<0.001$ \\
\hline SOFA score & $4.0(2.8,6.0)$ & $7.0(5.3,10.8)$ & $<0.001$ \\
\hline APACHE II score & $11.0(8.0,15.3)$ & $20.5(16.0,25.0)$ & $<0.001$ \\
\hline
\end{tabular}


Table 2 Logistic regression analysis of 28-day death in sepsis patients

\begin{tabular}{|l|l|l|l|}
\hline Predictor & OR & $\mathbf{9 5 \%} \mathbf{C l}$ & $\boldsymbol{P}$ \\
\hline Age & 1.046 & $1.020-1.074$ & 0.001 \\
Pct & 1.115 & $1.055-1.177$ & $<0.001$ \\
CRP & 1.016 & $1.011-1.021$ & $<0.001$ \\
Lac & 1.887 & $1.518-2.346$ & $<0.001$ \\
NLR & 1.054 & $1.032-1.076$ & $<0.001$ \\
SOFA score & 1.434 & $1.293-1.591$ & $<0.001$ \\
APACHE II score & 1.231 & $1.166-1.300$ & $<0.001$ \\
\hline
\end{tabular}

Table 3 Logistic regression analysis of SOFA combined with NLR for 28-day death in sepsis patients

\begin{tabular}{|l|l|l|l|l|l|}
\hline Predictor & $\boldsymbol{\beta}$ coefficient & SE & Wald statistic & $\boldsymbol{P}$ & OR \\
\hline SOFA & 0.376 & 0.057 & 43.079 & $<0.001$ & 1.456 \\
NLR & 0.056 & 0.012 & 22.223 & $<0.001$ & 1.057 \\
Constant & -4.327 & 0.467 & 85.872 & $<0.001$ & 0.013 \\
\hline
\end{tabular}

Table 4 Logistic regression analysis of SOFA composite indices for 28-day death in sepsis patients

\begin{tabular}{|l|c|l|r|}
\hline Predictor & OR & $95 \%$ Cl & \multicolumn{1}{c|}{$P$} \\
\hline Age combined with SOFA & 1.448 & $1.305-1.606$ & $<0.001$ \\
Pct combined with SOFA & 1.017 & $1.005-1.029$ & 0.005 \\
CRP combined with SOFA & 1.417 & $1.296-1.550$ & $<0.001$ \\
Lac combined with SOFA & 1.343 & $1.238-1.458$ & $<0.001$ \\
NLR combined with SOFA & 1.455 & $1.318-1.605$ & $<0.001$ \\
\hline
\end{tabular}

patients. Combined with SOFA and NLR, it was indicated that the predictor was SOFA+NLR $\times(0.056 / 0.376)$, namely, SOFA+NLR $\times 0.149$ (Table 3). The comparison of SOFA with NLR in the death group and the survival group showed that SOFA combined with NLR in the death group was significantly higher than the survival group (10.76 [7.80-13.24] vs 5.53 [3.59-7.24], $P<0.001$ ). Using the aforementioned predictor for logistic regression analysis, it was found that SOFA combined with NLR was a risk factor of death within 28 days in sepsis patients (OR 1.455, 95\% CI 1.318-1.605; $P<0.001)$. In addition, the prognostic efficacy of SOFA combined with age, Pct, CRP, and Lac was not as good as SOFA combined with NLR (Table 4).

\section{Predictive Value of Risk Factors of 28-Day Mortality in Patients with Sepsis}

Based on ROC analysis (Table 5 and Figure 2), age, Pct, CRP, Lac, NLR, SOFA, APACHE II, and NLR combined with SOFA could be used to predict the 28-day prognosis of sepsis patients. The AUC of NLR combined with SOFA (AUC $0.868,95 \%$ CI $0.824-0.911 ; P<0.001$ ) was higher than than that for SOFA score (AUC $0.791, Z=1.98>Z_{0.05}=1.96$; $P<0.05$ ) and NLR (AUC 0.721, $Z=3.48>Z_{0.05}=1.96, P<0.05$ ). The sensitivity and specificity of APACHE II and NLR combined with SOFA to predict 28-day outcomes for patients with sepsis were better than other indicators based on the Youden index. The $Z$ test was used to compare the AUC of APACHE II and NLR combined with SOFA, but yielded no significant difference between them $\left(Z=0.471<Z_{0.05}=1.96, P>0.05\right)$.

\section{Discussion}

Our study retrospectively analyzed the patients with sepsis admitted to the Emergency Medicine Clinical Research Center, and it was found that APACHE II score, SOFA score, and NLR combined with SOFA (SOFA+NLR $\times 0.149)$ could be used to predict the prognosis of sepsis patients within 28 days well, of which NLR combined with SOFA was optimal. 
Table 5 Predictive value of risk factors of 28-day mortality in patients with sepsis

\begin{tabular}{|l|c|c|c|c|c|c|c|c|c|c|}
\hline Predictor & AUC & 95\% CI & $\boldsymbol{P}$ & SE & Cutoff & $\begin{array}{c}\text { Sensitivity } \\
\text { (\%) }\end{array}$ & $\begin{array}{c}\text { Specificity } \\
\text { (\%) }\end{array}$ & $\begin{array}{c}\text { Youden } \\
\text { index }\end{array}$ & $\begin{array}{c}\text { Positive } \\
\text { likelihood } \\
\text { ratio }\end{array}$ & $\begin{array}{c}\text { Negative } \\
\text { likelihood } \\
\text { ratio }\end{array}$ \\
\hline Age & 0.645 & $0.573-0.717$ & $<0.001$ & 0.037 & 74.5 & 73.4 & 50.0 & 0.234 & 1.468 & 0.532 \\
Pct & 0.761 & $0.691-0.831$ & $<0.001$ & 0.036 & 0.405 & 67.2 & 78.6 & 0.458 & 3.140 & 0.417 \\
CRP & 0.746 & $0.677-0.816$ & $<0.001$ & 0.036 & 55.5 & 75.0 & 73.5 & 0.485 & 2.830 & 0.340 \\
Lac & 0.767 & $0.700-0.834$ & $<0.001$ & 0.034 & 2.05 & 56.3 & 80.3 & 0.366 & 2.858 & 0.544 \\
NLR & 0.721 & $0.651-0.790$ & $<0.001$ & 0.036 & 11.65 & 59.4 & 77.3 & 0.367 & 2.617 & 0.525 \\
SOFA & 0.791 & $0.729-0.853$ & $<0.001$ & 0.032 & 5.5 & 75.0 & 72.7 & 0.477 & 2.747 & 0.344 \\
APACHE II & 0.853 & $0.807-0.899$ & $<0.001$ & 0.023 & 14.5 & 84.4 & 73.1 & 0.575 & 3.138 & 0.213 \\
NLR & 0.868 & $0.824-0.911$ & $<0.001$ & 0.022 & 7.11 & 84.4 & 73.9 & 0.583 & 3.234 & 0.211 \\
combined & & & & & & & & & & \\
with SOFA & & & & & & & & & \\
\hline
\end{tabular}

Pct and CRP are commonly used to evaluate sepsis. Our results indicated that both Pct and CRP were able to predict 28-day mortality in sepsis patients. Consistently with our findings, Castellic et $\mathrm{al}^{5}$ found that in 150 sepsis patients, Pct and CRP levels were associated with severity of organ dysfunction. A study conducted by Rosanova et $\mathrm{al}^{6}$ on children with burns complicated by infection showed that Pct level had a sensitivity of $100 \%$ in predicting mortality within 30 days. Wang et $\mathrm{al}^{3}$ found that Pct was an independent risk factor that may affect the prognosis of patients with sepsis. Ganesan et $\mathrm{al}^{7}$ found that when compared with high-sensitivity CRP, CRP was a better predictor of neonatal sepsis because it has higher diagnostic specificity.

Lac is an anaerobic metabolite of cells, and increased levels indicate tissue hypoperfusion and increased anaerobic metabolism in patients with sepsis. It is also one of the prognostic indicators for patients with sepsis ${ }^{8,9}$ and an independent risk factor of death in patients with septic shock. ${ }^{10}$ In this study, Lac was a risk factor of death of sepsis patients, the same as in most studies. However, Lac is affected by many factors and needs to be evaluated in combination with other indicators.

NLR is an important inflammatory marker for the diagnosis and prognosis of sepsis patients. Zahorec et al ${ }^{11}$ conducted a prospective study of 90 ICU patients and found that NLR predicted the severity and prognosis of sepsis patients. Lorente et $\mathrm{al}^{12}$ found that NLR within 7 days after a diagnosis of sepsis was associated with the severity and mortality of sepsis. Chen et $\mathrm{al}^{13}$ studied 226 sepsis patients and found that the AUC of NLR in predicting the 28-day death rate in sepsis patients was $0.642(95 \%$ CI $0.556-0.728)$. Liu et $a^{14}$ also found that NLR was significantly correlated with the 28-day death rate of sepsis patients, similar to the results of our study.

In the Sepsis and Sepsis Treatment International Guidelines (Sepsis-3) developed in 2016, SOFA score has become an important diagnostic criterion for the diagnosis of sepsis ${ }^{1}$ and can predict the mortality of patients with sepsis (AUC $0.753,99 \%$ CI $0.750-0.757) .{ }^{15}$ In addition, Karakike et $a^{16}{ }^{16}$ demonstrated in 448 study cohorts and 199 validation cohorts that a decrease in SOFA score of $<25 \%$ within 7 days of admission was associated with increased mortality. Our study is the first to combine NLR with SOFA scores, and we found that NLR combined with SOFA (SOFA $+\mathrm{NLR} \times 0.149$ ) predicted the 28-day mortality of sepsis patients, and its AUC was 0.868 , higher than that of SOFA $(0.791)$ and NLR $(0.721)$. It could be seen from the Youden index that NLR combined with SOFA had high sensitivity and specificity.

The APACHE II score, which involves multiple factors, such as age, chronic health conditions, and acute physiology, can fully clarify the overall situation and predict the prognosis of patients. ${ }^{17}$ Higher APACHE II scores mean higher mortality. ${ }^{18}$ Our study found that APACHE II scores predicted the prognosis of patients well (AUC 0.853). While APACHE II scored at $\mathbf{1 4 . 5}$ or more, sensitivity and specificity for predicting 28-day mortality in sepsis patients were $84.4 \%$ and $73.1 \%$. However, APACHE II grading is based on 12 physiological indices (the worst value admitted to hospital within 24 hours), age and chronic health conditions to assess the severity of disease, 


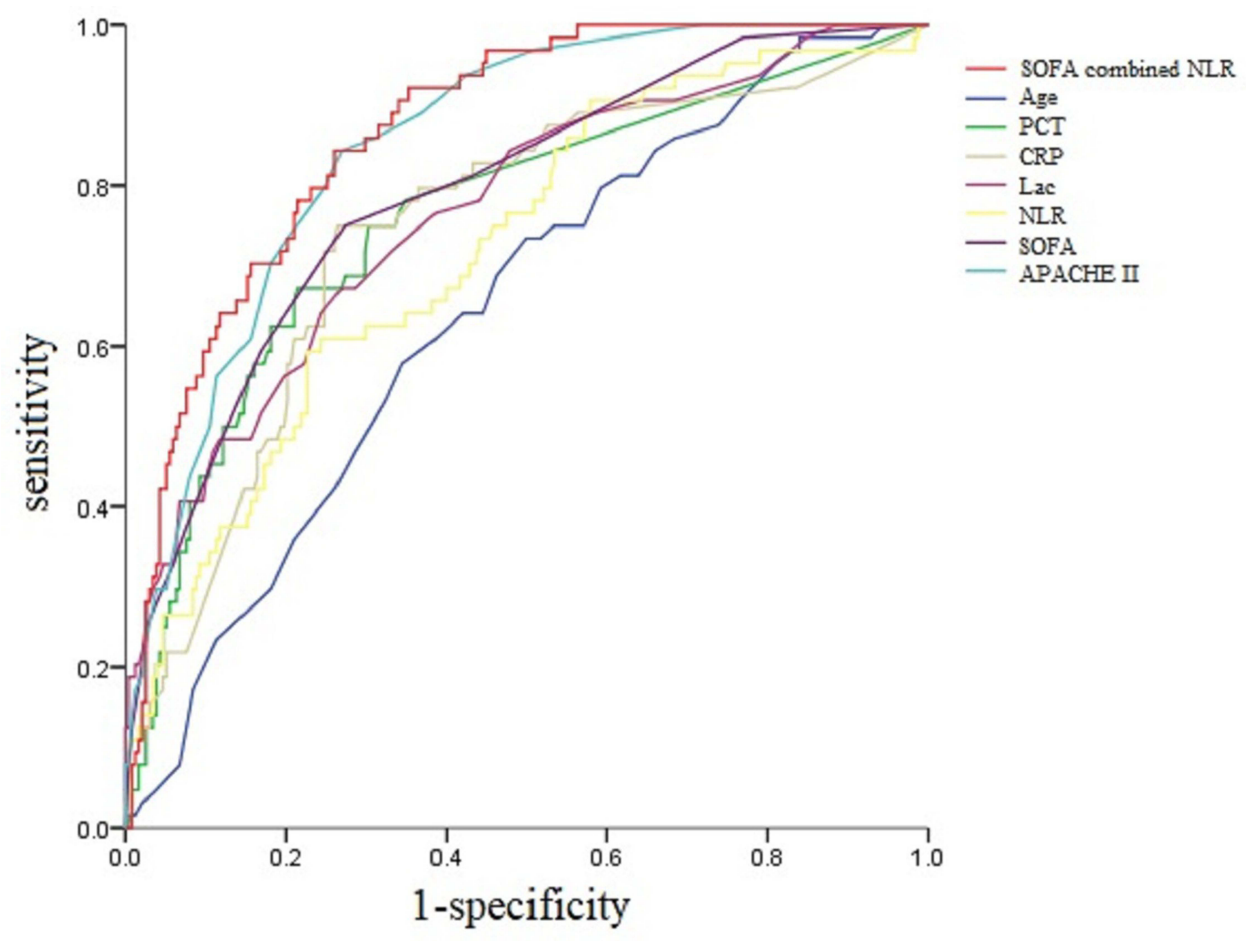

Figure 2 ROC for predicting 28-day mortality in sepsis patients.

Abbreviations: PCT, procalcitonin; CRP, C-reactive protein; Lac, lactic acid; NLR, neutrophil:lymphocyte ratio; SOFA, sequential organ failure assessment; APACHE, acute physiology and chronic health evaluation.

and needs lots of clinical data and laboratory tests, rendering it too complicated to obtain a specific rating initiallyand thus limiting its clinical application. Our study showed that the AUC of NLR combined with SOFA (SOFA $+\mathrm{NLR} \times 0.149$ ) in evaluating the 28-day mortality of sepsis patients was similar to that of APACHE II scoring. According to the $Z$ test, there was no significant difference between the AUC of NLR combined with SOFA and APACHE II scores, suggesting that the role of NLR combined with SOFA in evaluating disease status and outcomes in sepsis patients was consistent with the role of the APACHE II score. The assessment process of NLR combined with SOFA was simpler than that for APACHE II, which may provide a basis for early, rapid, and effective guidance of treatment.

There are still some limitations. Firstly, this was a single-center retrospective study with a medium sample size, and we lacked specific data on etiology and treatment, such as time to antibiotics, specific organ dysfunction, and percentage of ICU admissions. Secondly, our study lacks internal or external validation.

\section{Conclusion}

This study is the first to combine NLR with the SOFA score to identify risk factors in sepsis patients. NLR combined with SOFA (SOFA+NLR $\times 0.149$ ) was used to predict the prognosis of patients with sepsis within 28 days, which not only had high sensitivity and specificity but also maintained the speed and practicability of the scoring process. Also, predictive efficiency was consistent with the APACHE II score and better than other predictive indices.

\section{Abbreviations}

SOFA, sequential organ failure assessment; APACHE, acute physiology and chronic health evaluation; NLR, neutrophil: lymphocyte ratio; ROC, receiver-operating characteristic; Pct, procalcitonin; Lac, lactic acid; AUC, area under the curve; WBCs, white blood cells. 


\section{Data Sharing}

The data sets used and analyzed in the current study are available from the corresponding authors upon reasonable request.

\section{Ethics Approval and Consent to Participate}

This study protocols were in accordance with the principles of the Declaration of Helsinki and approved by the ethics committee of Beijing Chao-Yang Hospital (2016-7-4-1).

\section{Acknowledgments}

We sincerely thank the patients who participated in this study and the leadership and all staff of the Emergency Medicine Clinical Research Center, Beijing Chao-Yang Hospital.

\section{Funding}

The authors have no financial or proprietary interests in any material discussed in this article.

\section{Disclosure}

The authors report no conflicts of interest in this work.

\section{References}

1. Singer M, Deutschman CS, Seymour CW, et al. The third international consensus definitions for sepsis and septic shock (sepsis-3). JAMA. 2016;315(8):801-810. doi:10.1001/jama.2016.0287

2. Fleischmann C, Scherag A, Adhikari NK, et al. Assessment of global incidence and mortality of hospital-treated sepsis. Current estimates and limitations. Am J Respir Crit Care Med. 2016;193(3):259-272. doi:10.1164/rccm.201504-0781OC

3. Wang J, Wang J, Wei B. The diagnostic value of $\mathrm{Fe}(3+)$ and inflammation indicators in the death of sepsis patients: a retrospective study of 428 patients. Ther Clin Risk Manag. 2021;17:55-63. doi:10.2147/TCRM.S291242

4. Kumar S, Gattani SC, Baheti AH, et al. Comparison of the performance of APACHE II, SOFA, and mNUTRIC scoring systems in critically ill patients: a 2-year cross-sectional study. Indian J Crit Care Med. 2020;24(11):1057-1061. doi:10.5005/jp-journals-10071-23549

5. Castelli GP, Pognani C, Meisner M, et al. Procalcitonin and C-reactive protein during systemic inflammatory response syndrome, sepsis and organ dysfunction. Crit Care. 2004;8(4):R234-R242. doi:10.1186/cc2877

6. Rosanova MT, Tramonti N, Taicz M, et al. Assessment of C-reactive protein and procalcitonin levels to predict infection and mortality in burn children. Arch Argent Pediatr. 2015;113(1):36-41. doi:10.5546/aap.2015.36

7. Ganesan P, Shanmugam P, Sattar SB, et al. Evaluation of IL-6, CRP and hs-CRP as early markers of neonatal sepsis. J Clin Diagn Res. 2016;10(5): C13-C17.

8. Oh DH, Kim MH, Jeong WY, et al. Risk factors for mortality in patients with low lactate level and septic shock. J Microbiol Immunol Infect. 2019;52(3):418-425. doi:10.1016/j.jmii.2017.08.009

9. Liu Z, Meng Z, Li Y, et al. Prognostic accuracy of the serum lactate level, the SOFA score and the qSOFA score for mortality among adults with Sepsis. Scand J Trauma Resusc Emerg Med. 2019;27(1):51. doi:10.1186/s13049-019-0609-3

10. Zhao M, Duan M. [Lactic acid, lactate clearance and procalcitonin in assessing the severity and predicting prognosis in sepsis]. Zhonghua Wei Zhong Bing Ji Jiu Yi Xue. 2020;32(4):449-453. Chinese. doi:10.3760/cma.j.cn121430-20200129-00086

11. Zahorec R. Ratio of neutrophil to lymphocyte counts-rapid and simple parameter of systemic inflammation and stress in critically ill. Bratisl Lek Listy. 2001;102(1):5-14.

12. Lorente L, Martín MM, Ortiz-López R, et al. Association between neutrophil-to-lymphocyte ratio in the first seven days of sepsis and mortality. Enferm Infecc Microbiol Clin. 2020. doi:10.1016/j.eimc.2020.11.004

13. Chen J, Zhou X, Wang X, et al. Value of neutrophil to lymphocyte ratio in predicting the prognosis of patients with sepsis. Chin Gen Pract. 2017;20:533-537.

14. Liu Y, Zheng J, Zhang D, et al. Neutrophil-lymphocyte ratio and plasma lactate predict 28-day mortality in patients with sepsis. $J$ Clin Lab Anal. 2019;33(7):e22942. doi:10.1002/jcla.22942

15. Raith EP, Udy AA, Bailey M, et al. Prognostic Accuracy of the SOFA score, SIRS criteria, and qSOFA score for in-hospital mortality among adults with suspected infection admitted to the intensive care unit. JAMA. 2017;317(3):290-300. doi:10.1001/jama.2016.20328

16. Karakike E, Kyriazopoulou E, Tsangaris I, et al. The early change of SOFA score as a prognostic marker of 28-day sepsis mortality: analysis through a derivation and a validation cohort. Crit Care. 2019;23(1):387. doi:10.1186/s13054-019-2665-5

17. Knaus WA, Draper EA, Wagner DP, et al. APACHE II: a severity of disease classification system. Crit Care Med. 1985;13(10):818-829. doi:10.1097/00003246-198510000-00009

18. Schmidt DOA, Morello LG, Dalla-Costa LM, et al. Procalcitonin, C-reactive protein, albumin, and blood cultures as early markers of sepsis diagnosis or predictors of outcome: a prospective analysis. Clin Pathol. 2019;12:2632010X-19847673X. 


\section{Publish your work in this journal}

The International Journal of General Medicine is an international, peer-reviewed open-access journal that focuses on general and internal medicine, pathogenesis, epidemiology, diagnosis, monitoring and treatment protocols. The journal is characterized by the rapid reporting of reviews, original research and clinical studies across all disease areas. The manuscript management system is completely online and includes a very quick and fair peer-review system, which is all easy to use. Visit http://www.dovepress.com/testimonials.php to read real quotes from published authors.

Submit your manuscript here: https://www.dovepress.com/international-journal-of-general-medicine-journal 